\title{
THE INHIBITORY EFFECT OF RAMBUTAN PEEL EXTRACT TO THE Igf-1 AND Igf-1r EXPRESSION IN OBESE RATS VISCERAL FAT
}

\author{
Lestari, S.R. ${ }^{1,2}$, M.S. Djati ${ }^{3}$, A. Rudijanto ${ }^{4}$, Fatchiyah ${ }^{3}$ \\ ${ }^{1}$ Student of Biology, Department, Faculty of Science, Brawijaya University \\ ${ }^{2}$ Biology Departement, Faculty of Mathematics and Natural Sciences, State University of Malang \\ ${ }^{3}$ Biology Department, Faculty of Sciences, Brawijaya University \\ ${ }^{4}$ Endocrinology Department, Faculty of Medicine, Brawijaya University
}

\begin{abstract}
The activation of Insulin Like Growth Factor-1 (Igf-1) to its receptor (Igf-1r) plays an important role in the process of adipogenesis. The inhibition of Igf- 1 and its receptor at the early process is the precise step for the therapy of obesity. This study used obese rats treated with ellagic acid and Rambutan Peel Extract (RPE) $30 \mathrm{mg} / \mathrm{kgbw}$ dose. The Igf-1 and lgf-1r expression in visceral fat tissue was observed using immunofluorescence double staining, which confirmed later by western blotting. The results reveal that the use of RPE30mg/kg BW on obese rats model can provide inhibitory effect on the expression of igf- 1 and igf- $1 \mathrm{r}$ as in a normal rat. The expression of igf- 1 and its receptor highest in obese rats and the lowest expression in obese rats which were treated with ellagic acid. The reduced expression of igf- 1 and its receptor will reduce ERK $1 / 2$ and PPAR expression in adipogenesis signaling cascade and in turn inhibits adipogenesis.
\end{abstract}

Keywords: Igf-1, Igf-1r, Rambutan Peel Extract (RPE)

\section{INTRODUCTION}

High-carbohydrates and fats diets play an important role in stimulating the increasing of preadiposit proliferation and the expression of adipocyte's specific genes involved in the adipogenesis process. This kind of diet stimulates the release of growth factor, Igf-1 in particular (Marques et al., 2012; Viswakarma et al., 2010). Igf-1 plays an important role in the in vitro and in vivo changes of preadiposit into adipocytes, so that after the lgf-1 released, it will subsequently activate MAPK and triggered a series of genes reactions that play a role in adipogenesis (Gregoire et al.,1998; Holly et al., 2006; Kloting et al., 2008; Kondo et al., 2003).

The use of specific compound to inhibit the early process of adipocyte formation is the right step to reduce and avoid obesity. There are many specific compounds that can bind lgf1 or Igf- $1 \mathrm{r}$, which is widely used in the therapy of obesity. A particular and considered safe way to inhibit obesity (inhibit adipogenesis) is using herbal ingredients. World Health Organization (2012) described that people has increased their tendency, it is about $1 \%$ per year, to use herbal medicine. This data, trigger the exploration of tropic natural ingredients to be studied and used as the therapy or prevention against obesity.

Rambutan is a tropical fruit that grows well in Indonesia and the peel is considered as waste (Onkogi et al., 2007). Polyphenols, which consist of tannins, flavonoids and anthocyanins, is considered as the main consisted compounds on its peel. The purification of its phytochemical compounds showed that the peel is rich in geraniin, coraligin and ellagic acid (Thitilertdecha et al., 2010). The peel extract could be expected to inhibit igf-1 or igf-1r so that the cascade associated with adipogensis process could be inhibited as well. Many 
research described that the intensive research which is directly addressed to the adipocytes has the possibility for finding a potential drugs against obesity and overcome the insulin resistance (Ross et al.,1999).

The aims of this study was to know the RPE inhibition on igf- 1 and its receptors by measuring the igf- 1 and igf-1r using Immunofluorescence double stain and Western Blotting methods on obese male rats treated RPE $30 \mathrm{mg} / \mathrm{kgbw}$. The study was approved by ethical review committee of Brawijaya University Research Ethics Committee as a member of National Research Ethics Committee of Republic Indonesia.

\section{MATERIAL AND METHODS Material Preparations}

Male wistar rats, normal and obese, were obtained from d'Wistar, a laboratory animal provider, Bandung-Indonesia. Obese rats were fed with a high-calorie food after weaning until the $12^{\text {th }}$ week. The composition of normal food is $63 \%$ carbohydrate, $3 \%$ fat, $13 \%$ protein, $21 \%$ vitamin and mineral (manufactured by PT Comfeed, Indonesia) and the obese is $74 \%$ carbohydrate, $6 \%$ fat, $20 \%$ protein, vitamins, minerals and fiber $1 \%$ (manufactured by PT Phokphan, Indonesia). The criteria for obese rats was measured according to Lee Index (Campos et al., 2008). The rats' maintenance was performed in Biosciences laboratory, Brawijaya University. The dose of RPE used was $30 \mathrm{mg} / \mathrm{kgBW}$ (based on previous research) and ellagic acid (Sigma aldrigh, Catalogue number: E2550) with a dose $15 \mathrm{ig} / \mathrm{kgBB}$ was used as positive control. The treatment was performed over 12 weeks with RPE administration once every two days. After 12 weeks of treatment, the rats were dissected and the visceral fats were taken from the caudal side then washed with PBS pH 7,4. The fats weighed twice, 0,5 grams each. The first would be made into slides with immunofluorescence double staining and the last would be analyzed using western blotting method.

\section{Immunofluorescence Double Staining}

The control slides were stained using hematoxylin-eosin and the igf- 1 and igf-1r expression observed slides were stained using immunofluorescence double staining (modified from Bancroft \& Gamble, 2002). The slides were incubated overnight at $37^{\circ} \mathrm{C}$ to maximize the adhesion. Then, deparafination was conducted by xylol I and II for 5 minutes and the slides were soaked in $1 \mathrm{M} \mathrm{pH}$ 7.4 PBS three times for 5 minutes. The slides were subsequently immersed in $1 \mathrm{mM} \mathrm{pH} 6$ citrate buffer, heated in a microwave for 8 minutes, and incubated at room temperature for a few minutes, then soaked in PBS three times for 5 minutes. The slides were dried and put into a box that had been coated with tissue paper and sprayed with distilled water. A few drops of $2 \%$ BSA were sprinkled into the slides and incubated at room temperature for 1 hour. After that, the slides were washed with PBS three times for 10 minutes and were sprinkled using igf-1-mouse and igf-1r-rabbit (Lifespan Bioscience) primary antibody which was dissolved in $2 \%$ BSA (1:1500) then incubated at room temperature for 1 hour. The slides was washed again with PBS three times for 10 minutes and dried before sprinkled with the secondary antibody, Goat-antimouse IgG FITC and goatantirabbit IgG-Rhodamine (Santacruz Biotechnology) which was dissolved in 2\% BSA (1:2000). The slides were incubated for 1 hour at room temperature and washed with PBS 
three times for 10 minutes. Once dried, the immunofluorescence visualization by microspcope FSX-100, could be performed.

\section{Western Blotting}

The visceral fat tissue was pulverized using mortal and pistil, then added $500 \mathrm{il}$ extract buffer (1 mM PMSF in DMSO, $50 \mathrm{mM} \mathrm{pH} 7.4 \mathrm{KH}_{2} \mathrm{PO}_{4}, 0.5 \%$ Nonidet P.40, $\mathrm{ddH}_{2} \mathrm{O}$ ). The solution was centrifuged at $4^{\circ} \mathrm{C}$ and $10000 \mathrm{rpm}$ for 15 minutes. Protein from visceral fat was separated using $12.5 \%$ sodium dodecyl sulfate polyacrylamide gel (SDS-PAGE) and transferred to nitrocellulose membrane (Millipore, Belerica, MA) based on a wet transfer (Biorad). The primary antibody probe for igf-1-mouse and igf-1r-rabbit (Lifespan Bioscience) was dissolved in $2 \%$ BSA (1:1500) and incubated overnight $4^{\circ} \mathrm{C}$, then washed with TBS. The secondary antibody, anti-mouse $\lg G$ and lgG-antirabbit (Lifespan Bioscience) was dissolved in 2\% BSA (1:2000), incubated for 1 hour and given the HRP-conjugated secondary antibody substrate (Zymed), then incubated again for 1 hour. Western Blotting results were visualized using gel doc (Biorad system) and the measurement of the expressed proteins density was performed using quantity one software (Biorad).

\section{RESULTS AND DISCUSSION}

The immunofluorescence slides was used to measure the intensity of igf- 1 and igf-r expression. The results of immunofluorescence double staining in visceral fats tissue from normal-non-treated, obese-non-treated, ellagic acid, and RPE30 $\mathrm{mg} / \mathrm{kgBW}$ rats which was observed using microscope FSX-100. The observation suggests that the highest intensity of igf- 1 and igf-1r expression is in obese-NT rats compared with normal-NT, ellagic acid, and RPE30 mg/kgBW $(p<0,05)$. The igf- 1 expression intensity in normal-NT, obese-NT, ellagic acid, and RPE30mg/kgbw were $4.93 \mathrm{int} / \mathrm{cell}, 11.42 \mathrm{int} / \mathrm{cell}, 3.51 \mathrm{int} / \mathrm{cells}$ and $6.29 \mathrm{int} /$ cell respectively while the Igf-1r expression intensity in normal-NT, obese-NT, ellagic acid, and RPE30 mg/kgBW were $6.29 \mathrm{int} / \mathrm{cell}, 14.2 \mathrm{int} / \mathrm{cell}, 3.21 \mathrm{int} / \mathrm{cell}$ and $7.12 \mathrm{int} / \mathrm{cell} \mathrm{respec}-$ tively.

ANOVA test of igf- 1 and igf-1r expression among the treatments showed significant differences $(p<0,05)$. LSD test showed the differences in Igf- 1 and Igf- $1 \mathrm{r}$ expression from the highest to the lowest were obese-NT, RPE $30 \mathrm{mg} / \mathrm{kgBW}$, normal-NT and ellagic acid. The igf- 1 and igf-1r expression did not show any difference between among normal and obese rats treated withRPE30mg/kgBW, but showed a significant different among normal and obese rats with ellagic acid treatment.

Western Blotting results showed that the highest density of igf- 1 and igf-1r were in obeseNT compared to the other rats. Obese rats treated with ellagic acid showed the lowest density Igf- 1 than the others. The result of igf- 1 and igf- $1 \mathrm{r}$ density measurement showed that normal and obese rats treated with RPE30 $\mathrm{mg} / \mathrm{kgBW}$ seemed to have the same density.

Adipogenesis involves a complex series of processes, such as the process, which is triggered by a growth signal $(\mathrm{GH})$. GH stimulates the transcription of igf- 1 gene, which is required for adipocyte differentiation (Gregoire et al.,1998). Igf-1 initiates differentiation and stimulates cell on the clonal expansion of mitogenic effect. Also, the primary preadiposit from the clonal expansion secretes insulin growth factor-binding proteins (IGFBPs), which 
indicates that IGFBP is important in modulating the action of lgf- 1 in adipogenesis (Gregoire et al.,1998). Insulin Like Growth Factor-1 is a ligand that plays a role in insulin synthesis, mitogenic factor, cell growth and differentiation through several MAPKs pathway (Buehlmeyer et al., 2007). The activation of MAPKs pathway affects the expression of C/EBP and PPARã which becomes the key factor in adipogenesis (Lin et al.,2005). Igf-1 and igf-1r expression in obese rats showed a high value. The intake of high-calorie diet triggers the body to produce igf-1 and its receptor produced more. The high bond between Igf- 1 and its receptor causes the activation of Ras and stimulates the expression of ERK1/2 and MAPKs. MAPKs stimulation activates the key genes associated with adipogenesis process such as PPAR $\gamma$. Figure 2 shows that the density values of ERK $1 / 2$ is higher in obese rats compared to obese rats that were treated with ellagic acid and RPE30 $\mathrm{mg} / \mathrm{kg} \mathrm{BW}$.

Igf-1 and igf-1r expressions in obese rats, which were treated with ellagic acid showed the lowest value. Similar result showed by Boi et al. (2008) who described that ellagic acid inhibits adipocyte formation in 3T3-L1 cells and significantly inhibit PPAR $\gamma$ thus reducing lipid accumulation by $37 \%$. Ellagic acid a chemical compound produced synthetically and widely used as an anti-obesity that has the anti lipogenic properties and inhibit ERK1/2 (Min et al., 2012).

Treatment using RPE30 mg/kgBW decreased the expression of igf- 1 and igf- $1 \mathrm{r}$ almost similar to the value of normal rats. The decrease is thought to be caused by the content of phytochemical compounds in the crude extract of rambutan peel, which is able to bind to the Igf-1 or IGF-1r. One particular example is catechin, which is known as a potent compound that binds to Igf-1r (Firdausi et al., 2012). The tie between catechin and Igf-1r inhibits Igf-1 binding and its receptors. The decrease of Igf- 1 expression may be related to the ellagic acid content in the rambutan peel crude extract which has a similar properties to epigallocatechin-3-gallate (EGCG) green tea which can inhibit lgf-1 and Ras/Raf/MAPK signaling pathways (Unno et al., 2009). Several active compounds that block the activity of Igf1 and its receptor inhibits MAPKs signaling cascade resulting in low expression of ERK $1 / 2$ and PPAR $\gamma$. RPE30 $\mathrm{mg} / \mathrm{kg}$ BW decreased the expression of ERK1/2 and PPAR $\gamma$ approaching normal rats, but still higher compared with obese rats treated with ellagic acid. This result shows that RPE30 allegedly more effective than ellagic acid, due to the mixture of compounds contained in the crude extract of rambutan peel is expected to work synergic and antagonists causing a greater effect than pure ellagic acid treatment. RPE is an alternative treatment that can be used to overcome obesity, especially on the inhibition lgf- 1 and Igf-1r binding. Ross et al., described that the intensive research that directly addressed to the adipocytes has the possibility to provide a potential drugs against obesity and overcome the insulin resistance (Ross et al.,1999).

\section{CONCLUSION}

The increasing number of adipocytes mainly causes obesity, thereby inhibiting the formation of adipocytes is an important step to prevent obesity. Rambutan peel is a waste material yet has the potent to prevent the adipocytes formation. The results showed that the RPE has a potent in inhibiting adipogenesis by reducing the lgf- 1 and Igf-1r expression. The inhibition of lgf- 1 and its receptor will inhibit the cascade in MAPKs and PPARã pathway as 
the key genes for adipogenesis. This study is far from complete and we still need to do some further research related to the inhibition on adipogenesis cascade genes by observing the involved transcription factor.

\section{REFERENCES}

Buehlmeyer, K. 2007. IGF-1 gene expression in rat colonic mucosa after different exercise volumes. 434-440.

Campos, K. E., G.T. Volpato, I.M.P. Calderon, M.V.C. Rudge and D.C. Damasceno. 2008. Effect of obesity on rat reproduction and on the development of their adult offspring. Braziian Journal of Medical and Biological Research 41, 122-125.

Firdausi, L., M.R. Indra, and F. Fatchiyah. 2012. Binding Inhibition Between Igf-1r and Igf-1 by Catechin of Black Tea. The Journal of Tropical Life Science 2, 132-135.

Gregoire, F. M., Smas, C. M., and H.E.I.S. Sul. 1998. Understanding Adipocyte Differentiation. 78, 783-810.

Holly, J., M. Sabin, C. Perks, and J. Shield. 2006. Literature review. Matabolic Syndrome and Related Disorder 4: 43-50.

Kloting, N. 2008. Autocrine IGF-1 Action in Adipocytes Controls Systemic. Diabetes 57, 2074-2082.

Kondo, T. 2003. Knockout of insulin and IGF-1 receptors on vascular endothelial cells protects against retinal neovascularization. 111, 1835-1842.

Lin, J., M.A. Della-fera, C.A. Baile and A. Clifton. 2005. Inhibits Adipogenesis and Induces Apoptosis in 3T3-L1 Adipocytes.13.

Marques, B. G. 2012. Insulin-like growth factor I mediates high-fat diet-induced adipogenesis in Osborne-Mendel rats metabolism regulation Insulin-like growth factor I mediates highfat diet-induced adipogenesis in Osborne-Mendel rats. Physiol, Am J Integr, Regul 278, R654 - R662.

Min, S. Y. 2012. Cocoa polyphenols suppress adipogenesis in vitro and obesity in vivo by targeting insulin receptor. International Journal of Obesity 1-9.doi:10.1038/ijo.2012.85

Onkogi, S., C. Dhuangrat, S. Anuchpeed, S. Tachakitturungrod. 2007. Sombat Chowwanapoopahn Comparisson of Antioxidants Capasities and Cytotoxicities of Certain Fruit Peel. Jurnal of Medical Science 103.

Ross, S.E. 1999. Glycogen Synthase Kinase 3 Is an Insulin-Regulated C / EBP/ Kinase. 19, 8433-8441.

Thitilertdecha, N., A. Teerawutgulrag, J.D. Kilburn, N. Rakariyatham. 2010. Identification of Major Phenolic Compounds from Nephelium lappaceum L. and Their Antioxidant Activities. Molecule 15, 1453-1465.

Unno, T. 2009. Dietary tea catechins increase fecal energy in rats. Journal of nutritional science and vitaminology 55, 447-51.

Viswakarma, N. 2010. Coactivators in PPAR-Regulated Gene Expression. PPAR Research 2010, 1-22. 